\title{
Mechanical mitral valve modeling: Advancing the field through emerging science
}

\author{
Mersedeh Karvandi, ${ }^{2,}$, Saeed Ranjbar ${ }^{1}$, Seyed Ahmad Hassantash ${ }^{1}$ \\ ${ }^{1}$ Modarres Hospital, Institute of Cardiovascular Research, Shahid Beheshti University of Medical Sciences, Tehran, Iran \\ ${ }^{2}$ Taleghani Hospital, Shahid Beheshti University of Medical Sciences, Tehran, Iran
}

\section{Email address:}

mersedeh_karvandi@ipm.ir (M. Karvandi)

\section{To cite this article:}

Mersedeh Karvandi, Saeed Ranjbar, Seyed Ahmad Hassantash. Mechanical Mitral Valve Modeling: Advancing the Field through Emerging Science. International Journal of Medical Imaging. Vol. 2, No. 2, 2014, pp. 24-28. doi: 10.11648/j.ijmi.20140202.14

\begin{abstract}
Background: Successful mitral valve repair and replacement are dependent upon a full understanding of normal and abnormal mitral valve anatomy and function. The functional components of the mitral valve include: the left atrial wall, the annulus, the leaflets, the chordae tendineae, the papillary muscles, segments of the left ventricular myocardium. Abnormal anatomy or function of any one of these components can result in valvular dysfunction. We sought to assess the outcome of olden challenges of the mechanical behaviors of the mitral valve. Method: Much of our knowledge of abnormal mitral valve function is based on surgical and post-mortem studies while these studies are quantitative in some cases, they are limited by evaluation of valve anatomy in a fixed and nonfunctioning state. A more sophisticated analysis method is necessary to gain a full considerate of mitral valve function. Several groups attempted to model mitral valve anatomy and function by mathematical/physical equations. Result: Preliminary results concerning a different aspect of mitral valve leafletsbiomechanics, such as leaflets dynamics, displacements, thickness, stress and strain on leaflets. Conclusion: These data potentially allow the implementation of an image-based approach for patient-specific modeling of mitral valve leaflets. This approach could constitute the basis for accurate evaluation of mitral valve pathologic conditions and for the planning of surgical approaches.
\end{abstract}

Keywords: Mitral Valve, Mathematical Modeling, Finite Element Method, Stress-Strain of Leaflets, the Geometry of Mitral Valve, Fluid-Structure Interactions

\section{Introduction}

The left side of the heart accepts oxygenated blood at low pressure from the lungs into the left atrium. The blood then moves to the left ventricle which pumps it forward to the aorta to circulate the body. The heart's valves maintain the unidirectional flow of the blood through the heart, e.g. the mitral valve prevents regurgitation of blood from the left ventricle into the left atrium during ventricular systole and the aortic valve prevents blood flowing back from the aorta into the left ventricle during diastole. The principal fluid phenomena involved in the left ventricular diastolic flow are related to the presence of symmetric structures that develop with the strong jet that enters through the mitral valve (Reul et al., 1981; Saber et al., 2001) ${ }^{1-2}$. It is conjectured that the fibered structure has important effects on the function of the ventricle(Bacanni et al., 2003; Daebritz et al., 2003; Pierrakos and Vlachos, 2010) ${ }^{3-5}$.

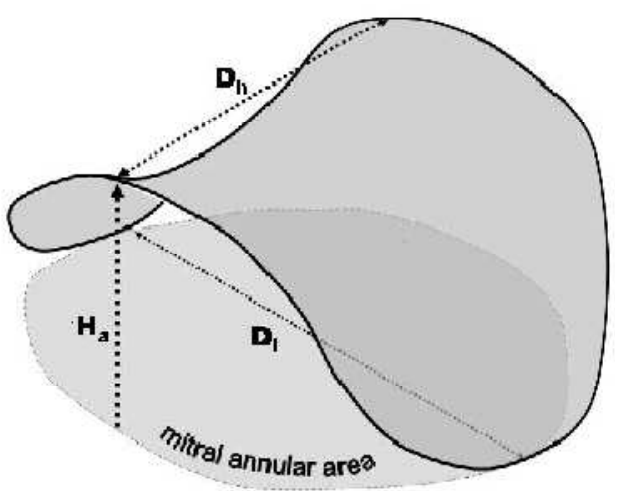

Figure 1.Schematic presentation of the non-planar, saddle-shaped mitral valve and itscharacteristic parameters: Dh-distance between high points; $\mathrm{Dl}$ - distance between lowpoints of the annulus; Ha- annular height. The mitral annular area is measured as anarea of the least squares plane

The human mitral valve is a complex anatomical structure consisting of two valve leaflets, an annulus, 
chordate tendineae, and two papillary muscles which are finger-like projections embedded into the underlying left ventricularmyocardium. The mitral annulus is a saddleshaped fibrous ring which seamlessly transitions into the two leaflets(Grashow et al., 2006; van Rijk-Zwikker et al., 1994) ${ }^{6-7}$ (Figure 1).The leaflets extend into the left ventricle where they are tethered to the papillary muscles via an intricate arrangement of chordae tendineae. The chordae tendineae consist of a complex web of chords that attach all over the leaflets of the valve.They prevent the prolapse of the valve leaflets at systole, and additionally assist in maintaining the geometry and functionality of the ventricle.

The papillary muscles play an important role and are believed to lengthen during isovolumetric contraction and shorten during ejection as well as during isovolumetric relaxation to maintain the chordae at the same deformation level, during opening and closure(Marzilli et al., 1980) ${ }^{8}$.

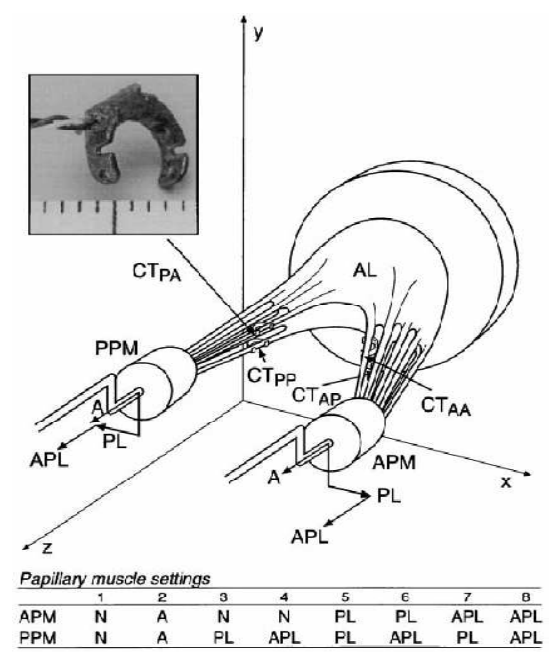

Figure 2.Schematic presentation of a simulator of the mitral valve

\section{Methods and Results}

The dynamic behaviour of porcine mitral valves has been studied in vitro by $\mathrm{He}$ et al. $(2003,2005)$ and Sacks et al. (2002) ${ }^{9-11}$. The Georgia Tech left heart simulator was used to measure surface strains in the valve leaflets under dynamic loading. The effect of the papillary muscle positions on the dynamic strains was also considered. This research provides important insight into the dynamic behaviour of the native mitral valve. Moreover, it enables a benchmark comparison for the performance ofthe mitral prosthesis. Both experimental and computational analysis can guide prosthesis designFigure 2.However, the advantage of a computational model is that material and geometric parameterscan be easily changed to determine an optimum design and it enables analysis that may be impractical experimentallyFigure 3.For example, stress and strain distributions can bepredicted; the mechanical interaction of the valve leaflets and fluid flow can be simulated in a physiologically realistic environment, e.g. a (prescribed) contracting ventricle. Unfortunately, computational modelling of the native mitral valve is difficult:the geometry of the chordae and leaflets is complex; the dynamic motion of the left ventricle causes a displacement of the papillary muscle base relative to the mitral annulus; the papillary muscles contract and relax during the cardiac cycle; the geometry of the mitral annulus is dynamic.

Furthermore, large deformation fluid-structure interactions are present during opening and closing phases. For these reasons, to date, only limited numerical research has been performed on mitral valves compared with aortic valves (Bellhouse, 1969; Ming and Zhen, 1986) 12-13.

Kunzelmann et al. (1993a, b) ${ }^{14}$ developed the first three-dimensional (3-D) finite-element model of the mitral valve usingANSYS. The open and closed mitral apparatus geometry was determined from resin casts of porcine mitral valves. This was used to construct a finite-element model that incorporated all essential anatomical components and regional tissue thicknessFigure 4.

A

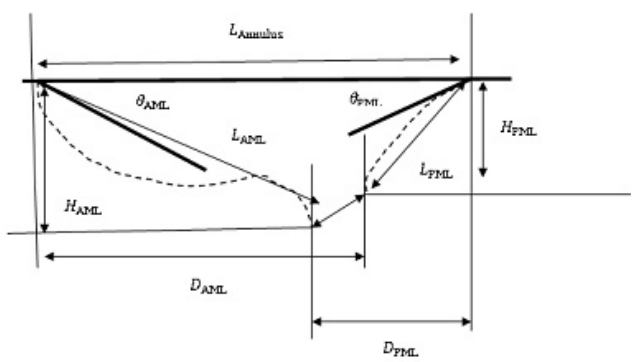

B

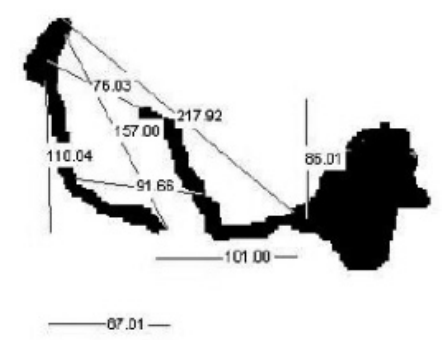

Figure 3.A) Parameters used to define the geometry of Mitral valve. B) The numerical calculation of geometric parameters of a mitral valve in MATLAB software

A

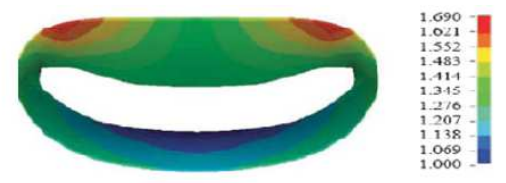

B

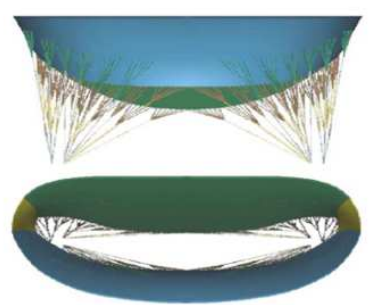

Figure 4.A) three-dimensional Finite element models of the mitral valve. It presented a model of the leaflets only to assess the effect of annular shape on leaflet curvature and stress.B) Geometry of mechanical model: leaflets, commissures, branched chords and multiple papillary insertions. 
The limitation of this model is that they assumed constant leaflet thickness, usednon-linear isotropic material models and did not include fluid flow.Anisotropic material properties were related to the collagen fibre orientation determined fromprevious studies. The closing phase of the mitral valve was simulated using a physiological timedependent loading andsolved using a quasi-static approach. The model was applied to give extensive further insight into the functioning andmalfunctioning of the mitral apparatus, e.g. to consider: the effects of chordae rupture, annular dilatation and leafletperforation (Kunzelmann et al., 1993a) ${ }^{15}$; the effect of replacing chordae tendineae with ePFTE suture (Reimink et al.,1996; Kunzelman and Cocharan, 1993) 16-17; annular dilatation (Kunzelman et al., 1997) 18 ; the effect of papillary muscleposition on mitral valve function (Cochran and Kunzelman, 1998) ${ }^{19}$; altered collagen concentration in mitral valve leaflets (Kunzelmanet al., 1998b) ${ }^{20}$. Note that in many of Kunzelman's studies, the papillary muscle tips (which the chordae attached to) werespatially fixed in position relative to a fixed mitral annulus. There is physiological justification for this: the subsequentcontraction and motion

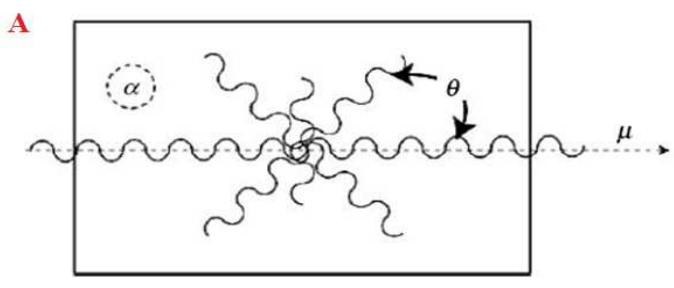

of the papillary muscles has the effect of fixing the distance between the papillary muscle tipsand the mitral annulus plane. Thus the lack of papillary muscle motion in the rigid left ventricular models does reflectthe constant papillary muscle tip to annular plane distanceFigure 4.

Salgo et al. (2002) ${ }^{21}$ presented a three-dimensional finite element model of theleaflets to assess the effect of annular shape on leaflet curvature and stress. Themicrostructure of mitral valve tissue is altered in response to stress resulting inchanges in tissue thickness, stiffness or both. In this model simulations of suchchanges increased tissue stiffness resulted in increased leaflet and chordae stresses, as well as reduced coaptation.

Einstein et al. (2010) ${ }^{22}$ recently developed the first computational model, which accounts for thecoupled fluidstructure interactions of the local blood flow and the mitral valve leaflets, during the closing phase of thecycle. The mitral leaflets were modelled as nonlinear membranes and the leaflet material was characterised as anorientated Gaussian distributed population of crimped collagen fibres embedded in an isotropic medium consisting ofglycoproteins and elastinFigure 5A.

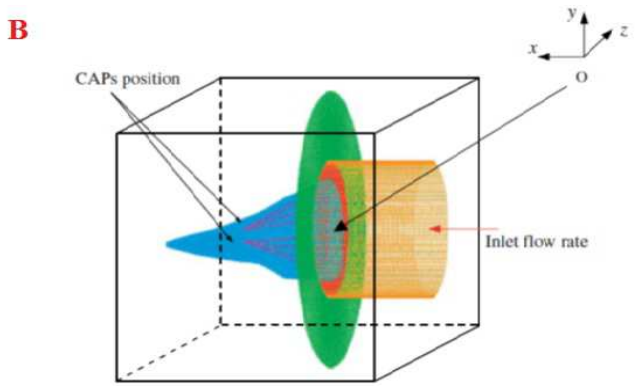

Figure 6.A) Mitral leaflet tissue material behavior is characterized as an oriented entangled population of crimped collagen fibers B)The chordae attachment points (CAPs)and the whole computational model of the mitral valve
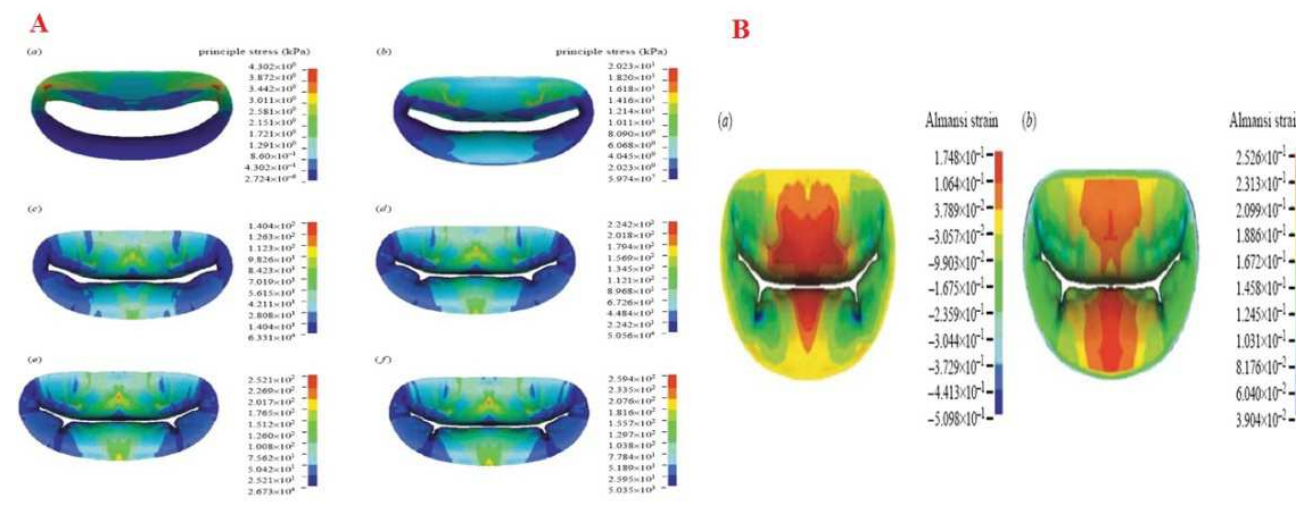

Figure 7.A) Time course of principle stress in the anterior and posterior leaflets in the normal model. Time: (a) 0.023, (b) 0.047, (c) 0.070, (d) 0.093, (e) 0.117 and (f) $0.140 \mathrm{~s}$. Red colour denotes the highest stress and dark blue the lowest B) (a) Circumferential and (b) radial strains at $0.14 \mathrm{~s}$ in normal model. Values with negative numbers are compressive strains.

The chordae were modeled as nonlinearly elastic tension cables. The papillary tips were fixedin position relative to the mitral annulus. Symmetry boundary conditions were applied on the septal-lateral midplane toreduce computational cost. The valve was immersed in a domain of Newtonian blood with ventricular and atrialpressure diagrams applied to the ventricular and atrial surfaces of the blood domainFigure 5B. Model predications showed excellentagreement with available transmitral flow, papillary force and first heart sound acoustic data.

Lim et al. (2010) ${ }^{23}$ recently developed the first asymmetric model of the mitral valve complex using ANSYS 5.7 for thewhole cardiac cycle. They observed that the motion is highly complex, and involves irregular 
twisting and skewing andthat the annulus and papillary tip motions are 3-D in the atrioventricular chamber. Thus asymmetric boundaryconditions are necessary for effective modelling of twisting or pulling of mitral valve. Distance tracings betweenultrasound crystals placed in the sheep mitral valve were converted into $3-\mathrm{D}$ coordinates to reconstruct an initialasymmetric mitral model and subsequent boundary conditions. The leaflets were modeled with linear isotropic materialproperties and uniform thickness. Nonlinear, real time left ventricular and aortic pressure loads were appliedsynchronously. The quasi-static deformations were determined over one time cycle. This study provided new insightinto the distribution of leaflet stress in the mitral valveFigure 6 :

In addition to the high leaflet stress that occurs during peak pressure loading, a prominent secondary peak (not observed previously) was observed during isovolumic relaxation of the ventricle. Note this model uses a quasistatic approach to model the valve deformation, i.e. it does not model the fluid-structure interactions.

\section{Recent Achievement}

Ranjbar S. Karvandi M. (2013) ${ }^{24-25}$ recently developed the first novelleft ventricular myocardial model

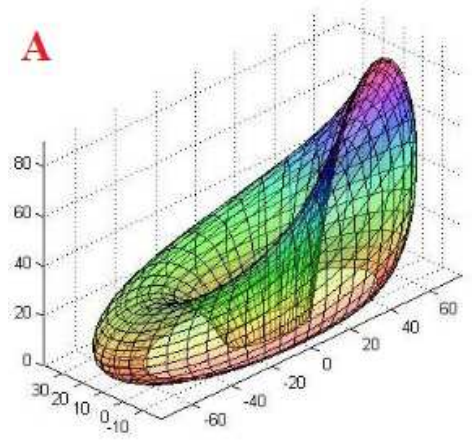

mathematically based on echocardiography, by MATLAB software and LSDYNA software in normal subjectsFigures 7 and 8, which dynamic orientation contraction (through the cardiac cycle) of every individual myocardial fiber could be created by adding together the sequential steps of the multiple fragmented sectors of that fiber. The left ventricular myocardial modeling of the heart shows that in normal cases myocardial fibers initiate from the posteriorbasal region of the heart, continues through the left ventricular free wall, reaches the septum, loops around the apex, ascends, and ends at the superior-anterior edge of left ventricle. This approach could overcome the limitations of previously proposed models and give new insight into the complex mitral valve function. This approach showsthe effect of fiber formation on left ventricular myocardium and mitral valve efficiency.

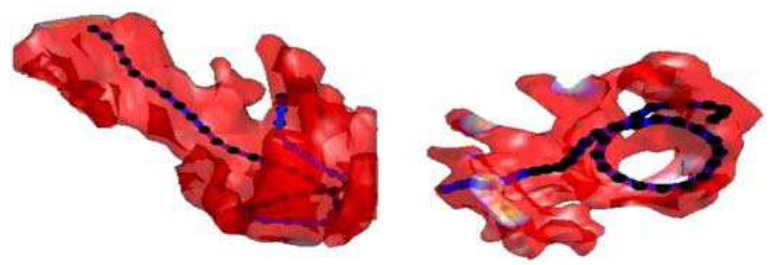

Figure 8. The rout of a fiber in the left ventricle

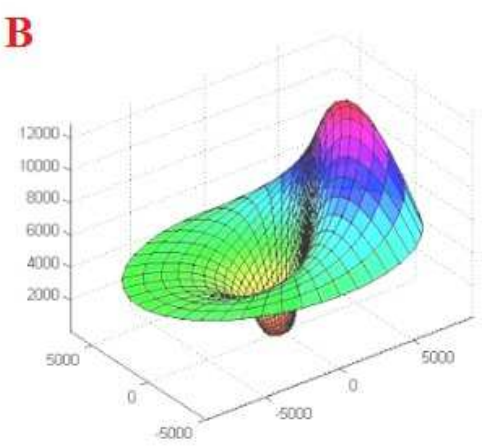

Figure 9.A) Impact of the direction of blood flow path to the left ventricular torsional deformation. B)Mathematical modeling of the left ventricle related to myocardial fiber paths.

\section{Disclosure}

There is no conflict of interest.

\section{References}

[1] Reul, H., Talukder, N., Muller, E.W. Fluid mechanics of the natural mitral valve. JBiomech1981; 14, 361-372.

[2] Saber, N.R., Gosman, A.D., Wood, N.B., Kilner, P.J., Charrier, C.L., Firmin, D.N.Computational flow modelling of the left ventricle based on in vivo MRI data: initialexperience. Ann Biomed Eng2001; 29, 275-283.

[3] Bacanni, B., Domenichini, F., Pedrizzetti, G. Model and influence of mitral valveopening during the left ventricular filling. J Biomech2003; 36, 355-361.
[4] Daebritz, S.H., Sachweh, J.S., Hermanns, B., Fausten, B., Franke, A., Groetzner, J,etal. Introduction of a flexible polymeric heart valve prosthesis with special design formitral position. Circulation 2003; 108, 134-139.

[5] Pierrakos, O., Vlachos, P.P. The effect of vortex formation on left ventricular fillingand mitral valve efficiency. J BiomechEng2006; 128, 527-539.

[6] Grashow, J.S., Yoganathan, A.P., Sacks, M.S. Biaxial stressstretch behavior of themitral valve anterior leaflet at physiologic strain rates. Ann Biomed Eng2006; 34, 315-325.

[7] vanRijk-Zwikker, G.L., Delemarre, B.J., Huysmans, H.A. Mitral valve anatomy andmorphology: relevance to mitral valve replacement and reconstruction. J Card Surg1994; 9, 255-261.

[8] Marzilli, M., Sabbah, H.N., Lee, T., Stein, P.D. Role of the papillary muscle inopening and closure of the mitral valve. Am J Physiol Heart CircPhysiol1980; 238,H348-H354. 
[9] He, Z.M., Sacks, M.S., Baijens, L., Wanant, S., Shah, P., Yoganathan, A.P. Effects ofpapillary muscle position on invitro dynamic strain on the porcine mitral valve. J HeartValve Dis 2003; 12, 488-494.

[10] He, Z.M., Ritchie, J., Grashow, J.S., Sacks, M.S., Yoganathan, A.P. In vitro dynamicstrainbehaviour of the mitral valve posterior leaflet. J BiomechEng2005; 127, 504511.

[11] Sacks, M.S., He, Z., Baijens, L., Wanant, S., Shah, P., Sugimoto, H, et al. Surfacestrains in the anterior leaflet of the functioning mitral valve. Ann Biomed Eng2002; $30,1281-1290$.

[12] Bellhouse, B.J., Bellhouse, F.H. Fluid mechanics of the mitral valve. Nature (London). 1969; 224, 615-618.

[13] Ming, L., Zhen, H.K. Study of the closing mechanism of natural heart valves. J ApplMath mechanics 1986; 7, 955964.

[14] Kunzelman, K.S., Cocharan, M.D. Finite element analysis of the mitral valve.JHeartValve Dis 1993; 2, 326-340.

[15] Kunzelman, K.S., Cochran, R.P., Verrier, E.D. Finite element analysis of mitral valvepathology. J Long Term Eff Med Implants 1993a; 3, 161-179.

[16] Reimink, M.S., Kunzelman, K.S., Cochran, R.P. The effect of chordalreplacementsuture length on function and stresses in repaired mitral valves: a finite element study.J Heart Valve Dis 1996; 5, 365-375.

[17] Kunzelman, K.S., Cocharan, M.D. Finite element analysis of the mitral valve. J HeartValve Dis 1993; 2, 326-340.

[18] Kunzelman, K.S., Reimink, M.S., Cochran, R.P. Annular dilatation increases stress inthe mitral valve and delays coaptation: a finite element computer model. J CardiovascSurg1997; 5, 427-434.

[19] Cochran, R.P., Kunzelman, K.S. Effect of papillary muscle position on mitral valvefunction: relationship to homografts. Ann ThoracSurg1998; 66, S155-S161.

[20] Kunzelman, K.S., Cochran, R.P., Murphree, S.S., Ring, W.S., Verrier, E.D., Eberhart,R.C. Differential collagen distribution in the mitral valve and its influence onbiomechanicalbehaviour. J Heart Valve Dis 1993b; 2, 236-244.

[21] Salgo, Ivan S., Gorman, Joseph H., Gorman, Robert C.Jackson Benjamin M. , BowenFrank W, et al. Effect of annular Shape on Leaflet Curvature in Reducing MitralLeaflet Stress. Circulation 2002; 106;711-717

[22] Einstein, D.R., Kunzelman, K.S., Reinhall, P.G., Nicosia, M.A., Cochran, R.P. Nonlinearfluid coupled computational model of the mitral valve. J Heart Valve Dis 2005; 14,376385 .

[23] Lim, K.H., Yeo, J.H., Duran, C.M.G. Three dimensional asymmetrical modeling of themitral valve: a finite element study with dynamic boundaries. J Heart Valve Dis 2005;14, 386-392.

[24] Ranjbar S. Karvandi M. Ajzachi M. System and method modeling left ventricle ofheart. US Patent 2013; patent number $8,414,490$.

[25] Ranjbar S. Karvandi M. S.A. Hassantash. A novel mathematical based software formodeling the left ventricular myocardium of heart [Abstract]Euroecho2012; P448 